\title{
Structure and Performance of Polyamide 6/Halloysite Nanotubes Nanocomposites
}

\author{
By Baochun GUO, ${ }^{*}$ Quanliang ZOU, Yanda LEI, and Demin JIA
}

\begin{abstract}
Halloysite nanotubes (HNTs) are chemically modified via silylation with 3-(trimethoxysilyl)propyl methacrylate. The modified HNTs (m-HNTs) are characterized by diffuse reflectance infrared Fourier transform (DRIFT) spectroscopy, solid state ${ }^{13} \mathrm{C}$ NMR spectroscopy, thermogravimetric analysis (TGA) and extraction experiment. It is showed that the silane has been effectively grafted onto HNTs surface and renders the hydrophobicity to m-HNTs. The nanocomposites consisting of polyamide 6 (PA6) and m-HNTs show significantly improved mechanical properties and heat distortion temperature, which are attributed to the covalent interfacial bonding and the excellent dispersion state of m-HNTs. M-HNTs are found to disperse individually into PA6 matrix. The nucleation effect by m-HNTs is verified by the lowered fold-surface free energy of PA6/ HNTs nanocomposites and the observation of polarized optical microscopy (POM). Both high cooling rate and high m-HNTs loading are beneficial to the formation of gamma-crystals of PA6. The polymorphism could be correlated to the heterogeneous nucleation effects of m-HNTs and the interfacial interactions between m-HNTs and PA6 matrix.

KEY WORDS: Polyamide / Halloysite / Nanocomposite / Mechanical Property / Crystallization /
\end{abstract}

Polyamide 6 (PA6) with high fatigue strength, low friction coefficient and high resistance to a wide spectrum of fuels, oils, and chemicals, is an important semicrystalline engineering plastics and finds its applications in automobiles, electrical appliances, construction materials, transportation materials and so on. However, the drawbacks such as limited mechanical strength and dimensional stability restrict its applications. ${ }^{1}$ Since the first introduction of PA6/clay nanocomposites in $1993,2,3$ they have drawn much attention due to their unprecedented performance compared with their microcomposite counterparts. The PA6 nanocomposites with inclusions of organic montmorillonite (O-MMT), ${ }^{4-6}$ carbon nanotubes $(\mathrm{CNTs})^{7-9}$ and attapulgite ${ }^{10,11}$ have been intensively investigated.

Halloysite nanotubes (HNTs) are a type of natural tubular nanoclay ${ }^{12-16}$ and have been recognized as the effective nanosized reinforcement of polymers for better mechanical properties and thermal properties. ${ }^{17-20}$ In the previous study, we have showed the reinforcing effects of the prinstine HNTs towords PA6. ${ }^{21}$ In the present work, we attempt to further improve the dispersion of HNTs and the interaction between HNTs and PA6 chains through the surface treatment of HNTs by silane of 3-(trimethoxysilyl)propyl methacrylate. The mechanical properties and heat distortion temperatures of the resulted nanocomposites are presented. The kinetic parameters regarding the chain folding activation energy during the nonisothermal crystallization are calculated. The polymorphism of the nanocomposites, which is dependent of the cooling rate of the melt and HNTs loading, is revealed. The performances such as mechanical properties, heat distortion temperature and polymorphism are correlated to the heterogeneous nucleation of HNTs and the interfacial interactions.

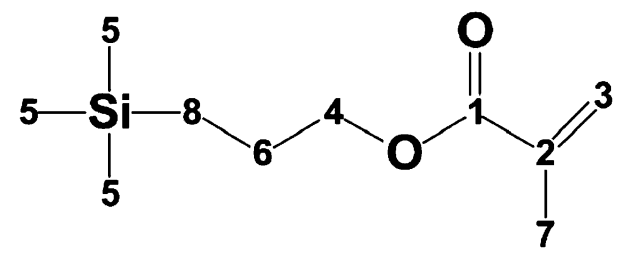

Scheme 1. Chemical formula of 3-(trimethoxysilyl)propyl methacrylate, MAPTS (numbers indicate the different chemical environments of carbon).

\section{EXPERIMENTAL}

\section{Materials}

Halloysite nanotubes (HNTs), mined in Yichang, Hubei Province of China were finely ground and purified according to the reported procedure. ${ }^{19}$ The purified HNTs were dried at $80^{\circ} \mathrm{C}$ for $12 \mathrm{~h}$. PA6 (ZISAMIDE TP6603), with molecular weight of 22700, was purchased from JI SHENG Co., Ltd, Taiwan and dried at $80^{\circ} \mathrm{C}$ for $12 \mathrm{~h}$ before blending with HNTs. 3-(Trimethoxysilyl)propyl methacrylate (MAPTS) 99\% (Scheme 1) was supplied by Fluka and used as received.

\section{Modification of HNTs}

MAPTS (20 g) was mixed for $30 \mathrm{~min}$ in $150 \mathrm{~mL}$ of ethanol $(95 \%)$ and the $\mathrm{pH}$ value of the solution was adjusted to 4 by acetic acid. The silane solution is kept under $40^{\circ} \mathrm{C}$ for half an hour, in order to fully hydrolyze the silane. HNTs $(5 \mathrm{~g})$ was then added into the hydrolyzed MAPTS solution, and the suspension was ultrasonicated for $30 \mathrm{~min}$ after mechanical stirring for $15 \mathrm{~min}$. The suspension was stirred for $1 \mathrm{~h}$ at $40^{\circ} \mathrm{C}$, and then the modified HNTs were washed by repeated 
centrifugation with pure ethanol, and finally dried at $80^{\circ} \mathrm{C}$ for $12 \mathrm{~h}$.

\section{Preparation of PA6/m-HNTs Nanocomposites}

The blends of PA6 and m-HNTs with designed composition were extruded with a twin-screw extruder and pelletized. The dried extrudates were injection moulded. The code of PA6/ m-HNTsX represents the PA6/m-HNTs nanocomposite with $\mathrm{X}$ parts $\mathrm{m}$-HNTs relative to 100 parts of PA6.

\section{Measurements and Characterizations}

Characterizations on m-HNTs. Fourier transform infrared spectra were recorded on a Bruker Vector 33 FT-IR spectrometer. The dried HNTs and m-HNTs samples were ground to fine particle, and the diffuse reflectance infrared Fourier transform (DRIFT) spectra were recorded against a $\mathrm{KBr}$ singlebeam background spectrum.

Solid-state ${ }^{13} \mathrm{C}$ NMR spectrum was recorded on a BRUKER AVANCE DSX $400 \mathrm{MHz}$ spectrometer operating at $100.6 \mathrm{MHz}$ for ${ }^{13} \mathrm{C}$, using the combination of cross-polarization and magic angle spinning (CP/MAS) methods. The spinning speed was set in the range of $6000 \mathrm{~Hz}$.

TGA of m-HNTs was carried out with TA Q5000 from room temperature to $600^{\circ} \mathrm{C}$ at a heating rate of $10^{\circ} \mathrm{C} / \mathrm{min}$ under $\mathrm{N} 2$ atmosphere.

The hydrophobicity of m-HNTs was verified by the extraction experiment. About $0.2 \mathrm{~g}$ HNTs and $0.2 \mathrm{~g}$ m-HNTs are separately added to two glass tubes. The tubes are filled with $10 \mathrm{~mL}$ toluene and followed by bath sonication for $10 \mathrm{~min}$. After further filling $10 \mathrm{~mL}$ deionized water, the tubes are subjected to further bath sonication for $10 \mathrm{~min}$. After standing, the observation on the retention of m-HNTs in solvent was made.

The ultrathin film was formed by dripping m-HNTs/alcohol suspension to water. The film was collected by a slide. The sample was dried and coated with a very thin layer of gold before the SEM observation. The instrument used in this study was a LEO1530 VP SEM machine. The voltage of the electron beam used for SEM observation is $10 \mathrm{kV}$.

Characterization on the PA6/m-HNTs Nanocomposites. Mechanical tests were conducted according to ISO 527: 1993, ISO 178: 1993 and ISO 180: 1993. The heat distortion temperature (HDT) was determined with a CEAST HDT/VICAT machine following ISO $75-2 /$ method $\mathrm{A}$. The stress for three-point bending is $1.8 \mathrm{MPa}$. The sample is preheated at $40^{\circ} \mathrm{C}$ for $2 \mathrm{~min}$ and heated at $2{ }^{\circ} \mathrm{C} / \mathrm{min}$. The HDT is the temperature under which the sample reaches the deformation of $0.34 \mathrm{~mm}$. The composite samples were unltramicrotomed (EM ULTRACUT UC, Leica) and observed with Philips Tecnai 12 TEM machine. The dynamic mechanical properties were analyzed using EPLEXOR $500 \mathrm{~N}$ with the three point bending model. The size of the samples is $30 \times 10 \times 4 \mathrm{~mm}$, and the temperature from -50 to $150{ }^{\circ} \mathrm{C}$. The heating rate is $3{ }^{\circ} \mathrm{C} / \mathrm{min}$ and the frequency is $5 \mathrm{~Hz}$. The crystalline morphologies were observed by using a polarized optical microscope (Olympus BX51 system). The samples were melted at $250{ }^{\circ} \mathrm{C}$ between two glass slides to obtain thin films with the thickness about $50 \mu \mathrm{m}$ and maintained at this temperature for $10 \mathrm{~min}$, after that the samples were cooled to $205^{\circ} \mathrm{C}$ at a constant cooling rate of $40^{\circ} \mathrm{C} / \mathrm{min}$, and maintained at this temperature for $30 \mathrm{~min}$, then cooled to ambient temperature at a constant cooling rate of $40{ }^{\circ} \mathrm{C} / \mathrm{min}$.

Differential Scanning Calorimetry (DSC) Procedures for the Evaluation of Kinetic Parameters. The non-isothermal crystallization of PA6/m-HNTs composites was analyzed by means of DSC TAQ 20. Before testing, all the samples were dried at $80^{\circ} \mathrm{C}$ for $12 \mathrm{~h}$ to remove moisture. All DSC analyses were performed under nitrogen purging. The non-isothermal crystallization of PA6/HNTs nano-composites was conducted as follows. The samples were heated from ambient temperature to $250^{\circ} \mathrm{C}$ at a heating rate of $40^{\circ} \mathrm{C} / \mathrm{min}$. The samples were kept for $5 \mathrm{~min}$ at this temperature to eliminate the thermal history. Then the samples were cooled to ambient temperature at a constant cooling rate of $2.5^{\circ} \mathrm{C} / \mathrm{min}, 5^{\circ} \mathrm{C} / \mathrm{min}, 10^{\circ} \mathrm{C} / \mathrm{min}$, $20^{\circ} \mathrm{C} / \mathrm{min}$, and $40^{\circ} \mathrm{C} / \mathrm{min}$. The thermograms were recorded for the calculation of the non-isothermal crystallization kinetics. The isothermal crystallization of PA6/m-HNTs nano-composites and subsequent melting behaviors were performed as follows: the sample was heated to $250{ }^{\circ} \mathrm{C}$ at a rate of $40^{\circ} \mathrm{C} / \mathrm{min}$ and held at this temperature for $5 \mathrm{~min}$ to eliminate any previous thermal history, and then jumped to the predetermined cryatallization temperature $\left(T_{\mathrm{c}}\right)$, ranging from 200 to $192^{\circ} \mathrm{C}$ in steps of $2{ }^{\circ} \mathrm{C}$ and was maintained at $T_{\mathrm{c}}$ for 30 min necessary for the DSC trace to return to the calorimeter baseline. The specimens were subsequently cooled to $30^{\circ} \mathrm{C}$ at a rate of $40^{\circ} \mathrm{C} / \mathrm{min}$ and then heated to $250^{\circ} \mathrm{C}$ at a rate of $10^{\circ} \mathrm{C} / \mathrm{min}$.

X-Ray Diffraction (XRD) for Polymorphism Study. The X-ray diffraction (XRD) patterns were recorded using the PANalytical X'pert PRO X-RAY Diffractometer. The CuK $\alpha$ radiation source was operated at $40 \mathrm{kV}$ and a current of $40 \mathrm{~mA}$. Patterns were recorded by monitoring those diffractions that appeared from $5^{\circ}$ to $30^{\circ}$. The scan speed was $1^{\circ} / \mathrm{min}$. The samples for XRD were prepared in the DSC chamber and the settings are the same as those in the non-isothermal crystallization in DSC experiments. Data of XRD were analyzed by use of origin 8.0 Peak Fitting Module, the values of $2 \theta$ of $\alpha$ phase crystals were estimated as $19.9^{\circ} \pm 0.1(\alpha 1)$ and $23.6^{\circ} \pm$ $0.1(\alpha 2)$ and the values of $2 \theta$ of $\gamma$-phase crystals and amorphous diffraction peak as $21.3^{\circ} \pm 0.1$ and $21.5^{\circ} \pm 0.2$ respectively. Each peak was modeled by using a Gaussian peak shape. The area of each peak was calculated, the crystallinity, and the proportions of each crystal form could be estimated. ${ }^{22,23}$ The deconvolution of the XRD curve is shown in Figure 1.

\section{RESULTS AND DISCUSSION}

\section{Characterizations on $\mathbf{m}-\mathrm{HNTs}$}

Our hypothesis is that the silane is covalently bonded to HNTs via the condensation between surface hydroxyls and the silane. This is confirmed by the DRIFT spectrum for m-HNTs. The comparison of DRIFT spectra of original HNTs and m- 


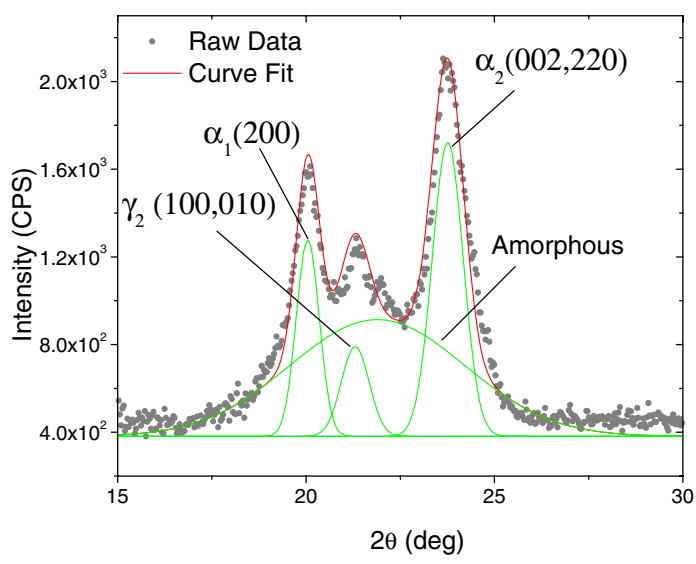

Figure 1. XRD pattern and the profile fitting technique used to deconvolute the amorphous and crystalline phases (PA6/m-HNTs2 sample at the cooling rate of $2.5^{\circ} \mathrm{C} / \mathrm{min}$ ).

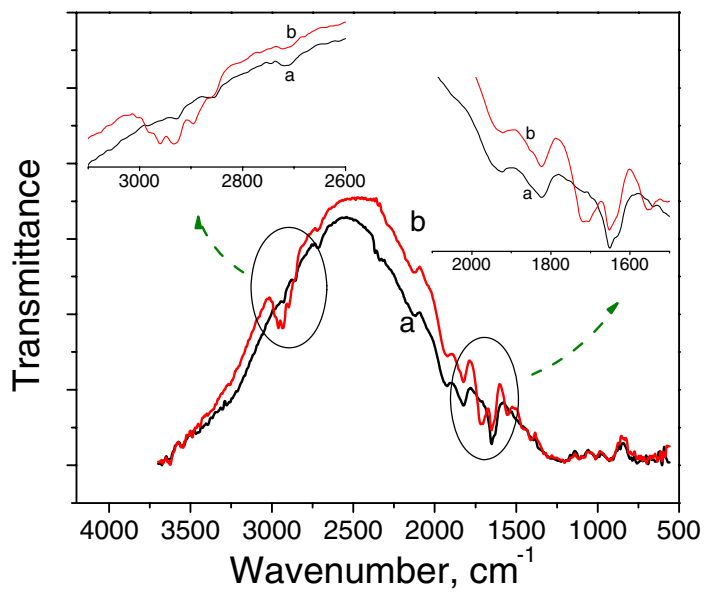

Figure 2. DRIFT spectra of of HNTs (a) and m-HNTs (b).

Table I. ${ }^{13} \mathrm{C}$ NMR chemical shifts for MAPTS and m-HNTs

\begin{tabular}{ccccccccc}
\hline Carbons & 1 & 2 & 3 & 4 & 5 & 6 & 7 & 8 \\
\hline MAPTS (ppm) & 167.39 & 136.74 & 125.13 & 66.63 & 50.57 & 22.25 & 18.34 & 5.49 \\
m-HNTs (ppm) & 167.69 & 137.61 & 125.10 & 67.25 & - & 23.23 & 18.97 & 9.82
\end{tabular}

HNTs are displayed in Figure 2. As shown in Figure 2, the spectral differences can be recognized. Compared with the spectrum of the original HNTs, several new peaks are found in that for m-HNTs. Peaks at 2960, 2933 and $2895 \mathrm{~cm}^{-1}$ are assigned to $v_{\mathrm{C}-\mathrm{H}}\left(\mathrm{O}-\mathrm{CH}_{2} \mathrm{CH}_{2}\right), v_{\mathrm{C}-\mathrm{H}}(\mathrm{Si}-\mathrm{R})$ and that at $1719 \mathrm{~cm}^{-1}$ is assigned to $v_{\mathrm{C}=\mathrm{O}}$ (methacryloxy group). ${ }^{24}$

The recorded ${ }^{13} \mathrm{C}$ NMR spectrum for $\mathrm{m}$-HNTs is shown in Figure 3. The assignments for all the carbon are made in Table I. The standard ${ }^{13} \mathrm{C}$ NMR spectrum is referred from the Spectral Database for Organic Compounds organized by National Institute of Advanced Industrial Science and Technology (AIST). ${ }^{25}$ It is shown that excepting C(5), all the carbon atoms are found in Figure 3, indicating fully hydrolysis of the silane and expected grafting of the silane on the HNTs. The hydrolysis of the silane is responsible for the slight shifts of the carbons towards to higher field.

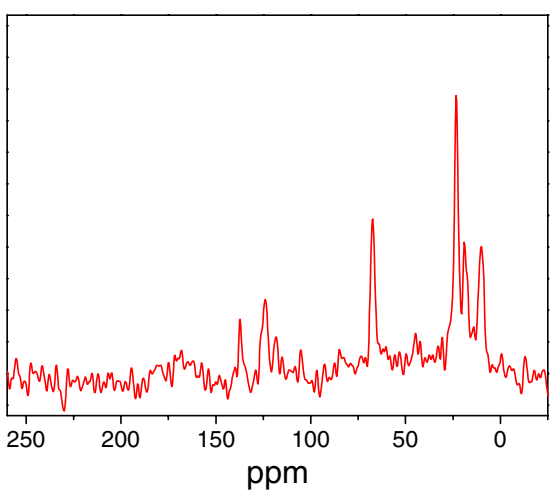

Figure 3. ${ }^{13} \mathrm{C}$ NMR spectrum of $\mathrm{m}-\mathrm{HNTS}$.

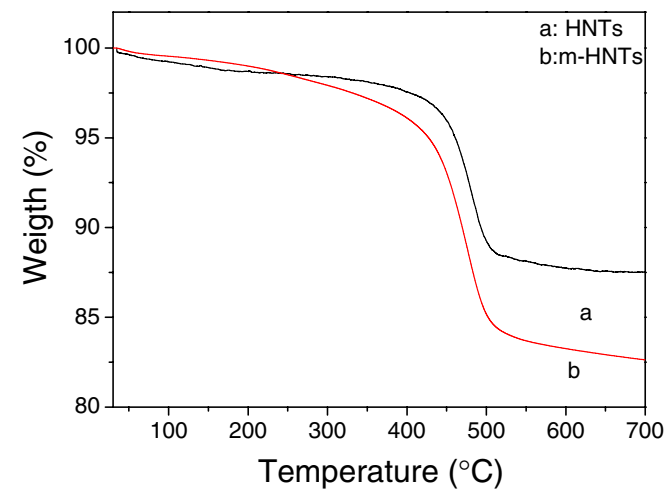

Figure 4. Thermal gravimetric analysis on HNTs (a) and m-HNTs (b).

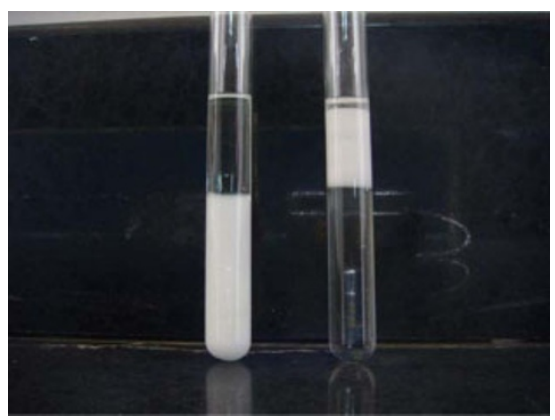

Figure 5. Comparison of the extraction of HNTs and m-HNTs in toluene/ water. left: HNTs; right: m-HNTs.

Figure 4 shows the weight losses of HNTs and m-HNTs. It is clear that the weight loss of m-HNTs is increased compared with that of the HNTs. The increased weight loss may be due to the decomposition of the grafted silane moiety or its condensates on the HNTs surface. The graft weight is calculated as 4.9 wt \% relative to the weight of HNTs.

The m-HNTs are found to be hydrophobic according to the extraction experiment. The result is presented in Figure 5. One can observe that HNTs is remained in water, and m-HNTs are remained in toluene. This indicates that the hydrophobic silane is successfully grafted on HNTs. Figure 6 is an ultrathin film formed by dipping alcohol suspension of m-HNTs on water. It is clear that the m-HNTs are laid flatly on the water surface 


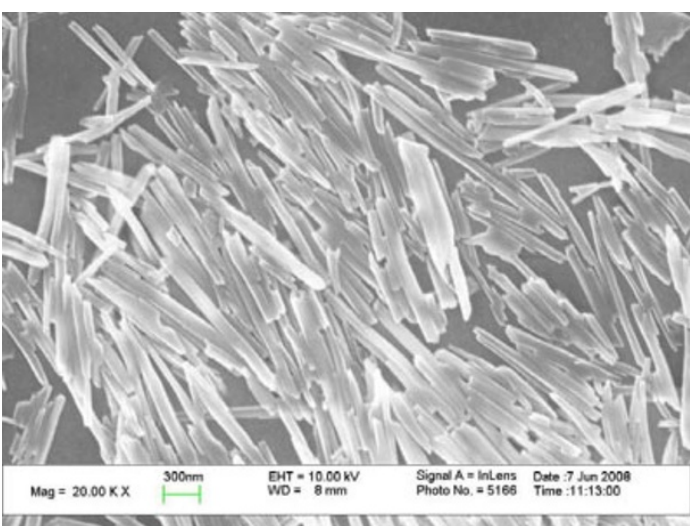

Figure 6. Ultra-thin film of m-HNTs (SEM photo).

Table II. Mechanical properties of PA6 and its nanocomposites

\begin{tabular}{cccccc}
\hline Sample codes & $\begin{array}{c}\text { Tensile } \\
\text { strength } \\
(\mathrm{MPa})\end{array}$ & $\begin{array}{c}\text { Flexural } \\
\text { strength } \\
(\mathrm{MPa})\end{array}$ & $\begin{array}{c}\text { Flexural } \\
\text { modulus } \\
(\mathrm{GPa})\end{array}$ & $\begin{array}{c}\text { Impact } \\
\text { strength } \\
\left(\mathrm{kJ} / \mathrm{m}^{-2}\right)\end{array}$ & $\begin{array}{c}\text { Heat } \\
\text { distortion } \\
\text { temperature } \\
\left({ }^{\circ} \mathrm{C}\right)\end{array}$ \\
\hline PA6 & 76.2 & 102.0 & 2.43 & 6.61 & 65.8 \\
PA6/m-HNTs2 & 85.2 & 117.2 & 2.92 & 6.30 & 76.8 \\
PA6/m-HNTs5 & 88.0 & 123.9 & 3.24 & 6.69 & 108.3 \\
PA6/m-HNTs10 & 92.5 & 131.1 & 3.79 & 7.05 & 114.1 \\
\hline
\end{tabular}

almost as a monolayer, suggesting no intertubular entanglements in m-HNTs.

\section{Performance of PA6/m-HNTs Nanocomposites}

Table II shows the effects of m-HNTs content on the mechanical properties of the PA6 nanocomposite. It can be seen from Table II, with increasing m-HNTs content, the tensile strength, flexural strength and flexural modulus of the composites are substantially increased, while the impact strength is increased slightly. For example, when $10 \mathrm{phr}$ (about $9 \mathrm{wt} \%$ ) of HNTs are incorporated, the tensile strength, flexural strength and flexural modulus were increased by $21 \%, 29 \%$ and $56 \%$ respectively. Compared to the unmodified HNTs, ${ }^{21} \mathrm{~m}-$ HNTs are more effective for improving the mechanical properties of PA6. There are two origins for this. On the one hand, because the surface hydroxyl and silicon-oxygen has been covered by the silane, the hydrogen bonds among the tubes are considerably eliminated and consequently the mHNTs are more uniformly dispersed in PA6 matrix. This can be confirmed from the TEM photographs of the PA6/m-HNTs nanocomposites (Figure 7). From Figure 7 it can be observed that, regardless of the content, m-HNTs are dispersed individually and there are almost no aggregates. On the other hand, the silane coupling agent MAPTS of $\gamma$-methacrylopropyloxy of the silane may covalently bonded with the amine ends of the PA6 chains via Michael addition, resulting greatly improved interfacial bonding between PA6 and HNTs. The combination of individually dispersed inorganic nanotubes and the covalently bonded interface renders excellent mechanical properties for the PA6/m-HNTs nanocomposites.
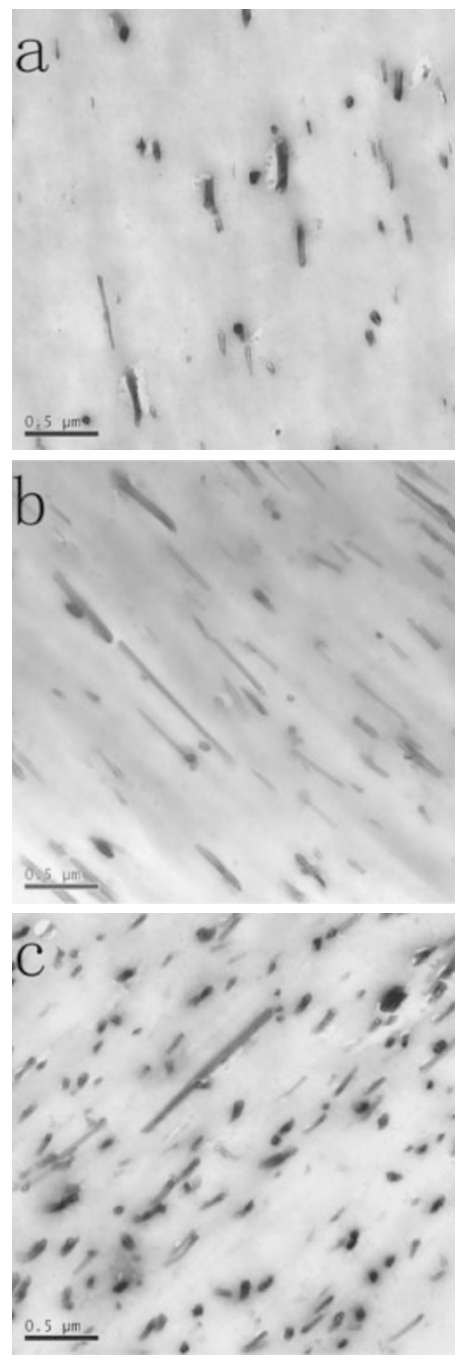

Figure 7. TEM photographs of PA6 nanocomposites (a) PA6/m-HNTs2, (b) PA6/m-HNTs5, (c) PA6/m-HNTs10.

The heat distortion temperature results are also shown in Table II, we can see that the the HDT of PA6 is greatly improved by the incorporation of m-HNTs. For example, when $10 \mathrm{phr}$ (about $9 \mathrm{wt} \%$ ) of HNTs are incorporated, improvement above $48{ }^{\circ} \mathrm{C}$ in HDT is achieved. The largely improvement in HDT is attributed to the effective load transferring from the matrix to HNTs via the strong interfacial interactions.

Figure 8 shows the temperature dependences of storage modulus and tangent of loss angle (Tan $\delta$ ) of PA6 and the PA6/ m-HNTs nanocomposites. From Figure 8(a), we can see that with the increase in HNTs content, the storage modulus of the PA6 nanocomposite is increased significantly. For instance, in PA6/m-HNTs, the storage moduli at glass state $\left(-25^{\circ} \mathrm{C}\right)$ and rubber state $\left(100^{\circ} \mathrm{C}\right)$, are increased by $46.6 \%$ and $66.4 \%$. The simultaneous increases in glassy modulus and rubbery modulus further indicate the strong interfacial interaction in the nanocomposites. From Figure 8(b), we can see that the $T_{\mathrm{g}}$ of the nanocomposites $\left(67.9^{\circ} \mathrm{C}\right.$, independent of HNTs content) is slightly lower than that for the neat PA6 $\left(70.5^{\circ} \mathrm{C}\right)$. In addition, the tangent delta value of the nanocomposite is consistently 


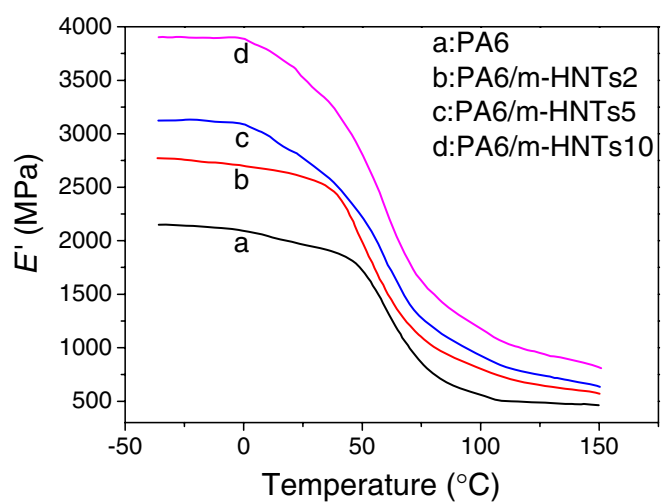

(a)

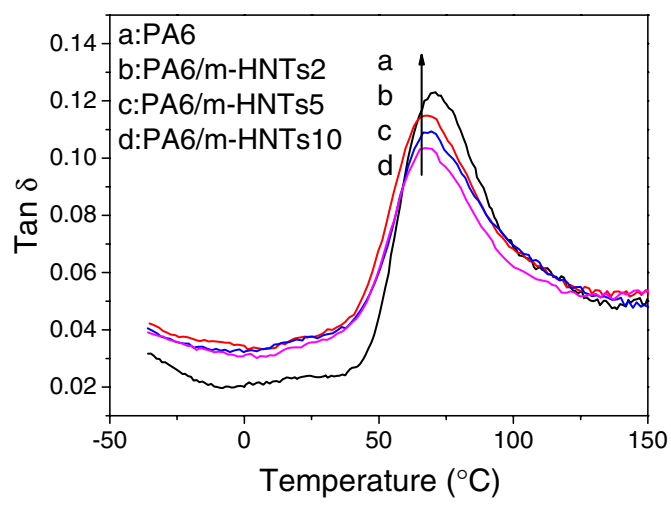

(b)

Figure 8. DMA curves of PA6 and PA6/m-HNTs nanocomposites (a. storage modulus; b. Tan $\delta$ ).

decreased with the HNTs content. The decrease in $T_{\mathrm{g}}$ may be attributed to the decreased intermolecular hydrogen bonding due to the HNTs. The change in $T_{\mathrm{g}}$ is different from those in the carbon nanotubes $(\mathrm{CNTs})^{26}$ or 2-dimensional layered MMT reinforced PA6 nanocomposites. ${ }^{27}$ In the CNTs incorporated systems, the CNTs showed weak interfacial interaction with PA chain, resulting unchanged $T_{\mathrm{g}}$. In the PA6/MMT nanocomposites, due to the great restriction of chain mobility by the MMT sheet, the $T_{\mathrm{g}}$ of PA was found to be higher. Due to chemical linkages between m-HNTs and PA6 chain, the segmental movement of PA6 chain is restricted, resulting lowered loss in glass transition region.

Evaluation of Kinetic Parameters for Non-Isothermal Crystallization via Nonlinear Isoconversional Method

The theoretical background has previously reported. ${ }^{28-31}$ The dependences of immediate crystallization activation energy $\left(E_{\alpha}\right)$ on the crystallinity $(\alpha)$ for nylon 6 and its nanocomposites are presented in Figure 9. The dependences of $\alpha$ on temperature are also integrated in Figure 9. Because there are five cooling rates, a given $\alpha$ corresponds to five temperatures and the average of the five temperatures is used for constructing the relationship between $\alpha$ and $T$ in Figure 9. From Figure 9, it can be seen that, for neat PA6 and all the nanocomposites, the absolute value of $E_{\alpha}$ decreases as $\alpha$

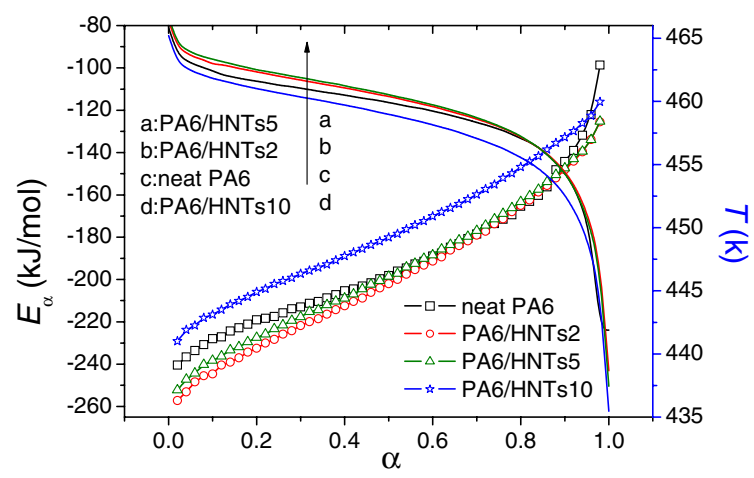

Figure 9. Dependence of the effective activation energy on the relative extent of crystallization. The solid line represents the variation of the average temperature with the relative extent of crystallization.

increases and at the same $\alpha$, the absolute value of $E_{\alpha}$ of the nanocomposites increases at first as m-HNTs loading increases, and then decreases as m-HNTs loading increases. When the concentration of m-HNTs is $10 \mathrm{phr}$, the nanocomposite possesses even lower absolute $E_{\alpha}$ value compared with the neat PA6. The crystallization involves nucleation and grain growth stages. The measured $E_{\alpha}$ is the overall energy consisting of that for nucleation and that for grain growth. If the grain growth is restricted, the activation energy for the grain growth will be higher. If additional sites are provided, the activation energy for the nucleation will be lower. There are two aspects of impact of m-HNTs on the crystallization of PA6. On the one hand, m-HNTs act as heterogeneous nuclei and promote the nucleation; On the other hand, the strong interaction between m-HNTs and PA6 chains limits the mobility of PA6 chain, resulting difficulty in the grain growth. When HNTs loading is lower, the crystallization process mainly is controlled by the grain growth, because less nuclei are generated and there is larger space for grain growth; When the HNTs content is high, the crystallization process is mainly under the control of nucleation, since much nuclei are generated and excessive nucleation leads to a dramatic reduction in space for grain growth. Thus the dependence of absolute $E_{\alpha}$ on m-HNTs loading exhibits a trend of increasing first and then rapidly decreasing.

We could establish the relationship between $E_{\alpha}$ and $T$ from data in Figure 9 and this is depicted in Figure 10. the relationship between $E_{\alpha}$ and $T$ could be described by eq (1).

$$
E_{\alpha}(T)=U^{*} \frac{T^{2}}{\left(T-T_{\infty}\right)^{2}}+K_{\mathrm{g}} R \frac{\left(T_{\mathrm{m}}^{0}\right)^{2}-T^{2}-T_{\mathrm{m}}^{0} T}{\Delta T^{2} T}
$$

Where $U^{*}$ is the activation energy of the segmental jump, $\Delta T=T_{\mathrm{m}}^{0}-T$ is the under-cooling, $T_{\infty}$ is a hypothetical temperature at which viscous flow ceases (usually ${ }^{29}$ taken $30 \mathrm{~K}$ below the glass transition temperature, $T_{\mathrm{g}}$ ). The kinetic parameter $K_{\mathrm{g}}$ has the following form:

$$
K_{\mathrm{g}}=\frac{n b \sigma \sigma_{\mathrm{e}} T_{\mathrm{m}}^{0}}{\Delta h_{\mathrm{f}}^{0} k_{\mathrm{B}}}
$$




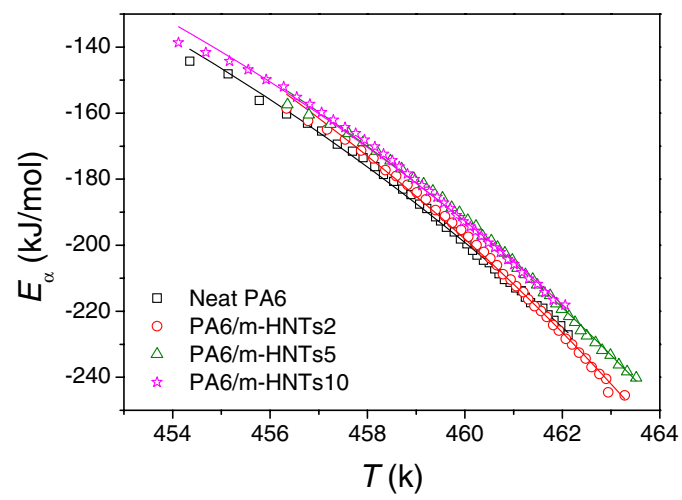

Figure 10. Dependence of the effective activation energy on average temperature. The solid lines represent fits of eq (1).

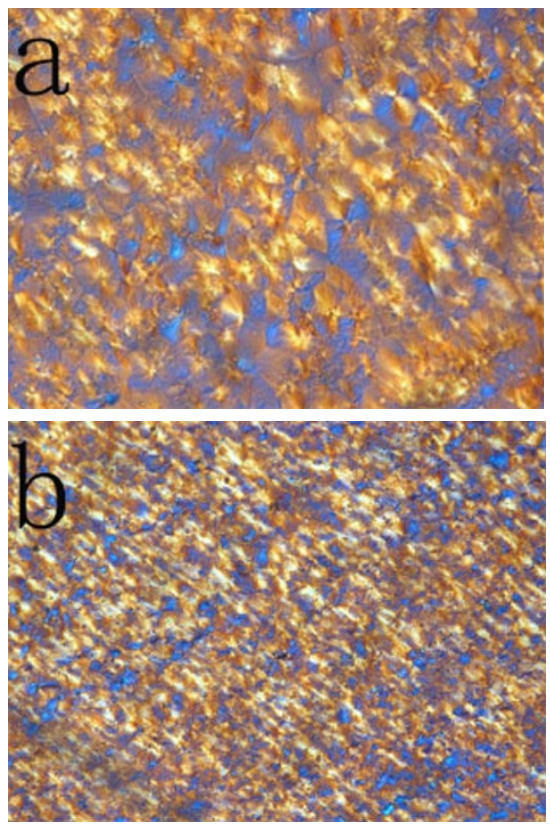

Figure 11. POM micrographs of (a) neat PA6, and (b) PA6/m-HNTs5.

Where $n$ takes the value 4 for crystallization regime I and III, and 2 for regime II. $\mathrm{b}$ is the distance between two adjacent fold planes; $\sigma$ and $\sigma_{\mathrm{e}}$ are the lateral and folding surface free energy, respectively $T_{\mathrm{m}}^{0}$ is the equilibrium melting temperature, $\Delta h_{\mathrm{f}}^{0}$ is the heat of fusion per unit volume of crystal, $k_{\mathrm{B}}$ is the Boltzmann constant. In previous paper, ${ }^{28}$ we ascribe PA6 crystallization behavior to Regime I. In view of the limited loading (below $10 \mathrm{wt} \%$ ), we still treat the crystallization of PA6/m-HNTs nanocomposites in Regime I.

Using the software Origin 8.0, the data in Figure 11 is fitted with eq (1), and $U^{*}$ and $K_{\mathrm{g}}$ could be calculated. With eq (2), the values of $\sigma \sigma_{\mathrm{e}}$ could be calculated. The fitting results are summarized in Table III.

The product of $\sigma \sigma_{\mathrm{e}}$ can be split by using the empirical relationship: ${ }^{32}$

$$
\sigma=0.11 \Delta h_{\mathrm{f}}^{0} \sqrt{a b}
$$

Table III. Kinetic data for non-isothermally crystallization of neat PA6 and its nanocomposites

\begin{tabular}{cllll}
\hline Sample & Neat PA6 & PA6/HNTs2 & PA6/HNTs5 & PA6/HNTs10 \\
\hline$U^{*}(\mathrm{~J} / \mathrm{mol})$ & 5485.58 & 7300.81 & 4956.79 & 5089.25 \\
$K_{\mathrm{g}}\left(10^{5} \mathrm{~K}^{2}\right)$ & 1.85 & 1.80 & 1.50 & 1.62 \\
$R^{2}$ & 0.99752 & 0.99686 & 0.99736 & 0.99664 \\
$T_{\mathrm{m}}^{0}(\mathrm{~K})$ & 509.62 & 507.75 & 506.04 & 507.75 \\
$\sigma \sigma_{\mathrm{e}}\left(10^{-4} \mathrm{~J}^{2} / \mathrm{m}^{4}\right)$ & 1.70 & 1.66 & 1.39 & 1.49 \\
$\sigma_{\mathrm{e}}\left(10^{-3} \mathrm{~J} / \mathrm{m}^{2}\right)$ & 5.13 & 5.01 & 4.20 & 4.50 \\
\hline
\end{tabular}

Which allows evaluation of lateral surface free energy from the parameters $a$ and $b$ of the PA6 unit cell., ${ }^{1,33}$ The $\sigma$ was calculated as $3.3 \times 10^{-2} \mathrm{~J} \cdot \mathrm{m}^{-2}$. The values for $\sigma_{\mathrm{e}}$ were also summarized in Table III. From Table III, it is clear that the nanocomposites possess lower $\sigma_{\mathrm{e}}$ compared with the neat PA6, indicating the heterogeneous nucleation by m-HNTs.

The nucleation effect of m-HNTs could also be indicated by the POM observations. Figure 11 shows the the POM photographs for PA6 and the PA6/m-HNTs nanocomposites. It can be seen that compared with the neat PA6, the Maltese cross in the nanocomposite is much smaller and the interface among the crystals is fuzzier, suggesting the heterogeneous nucleation by m-HNTs.

Effects of Cooling Rate on the Crystallinity and Polymorphism

Figure 12 shows the XRD curves of the neat PA6 and the PA6/m-HNTs nanocomposites crystallized at variable cooling rate. The diffractions for $\alpha$ modification are found at $2 \theta$ of $19.9^{\circ}$ and $23.7^{\circ}$, while the diffraction for $\gamma$ modification is characterized at $2 \theta$ of $21.3^{\circ} .^{34,35}$ The diffraction at $2 \theta$ of $24.67^{\circ}$ in Figure 12(d) is attributed to HNTs. It can be seen from Figure 12, as the cooling rate increases, the intensity for the diffraction at $21.3^{\circ}$ gradually increases, suggesting increasing $\gamma$-crystalline content in all the samples. Compared with the neat PA6, even at low cooling rate $\left(2.5^{\circ} \mathrm{C} / \mathrm{min}\right)$, clear $\gamma$ crystalline diffraction is found in the PA6/m-HNTs nanocomposite, indicating that both high cooling rate and m-HNTs facilitate the formation of $\gamma$-crystals.

According to XRD data, the crystallinities of the neat PA6 and its nanocomposites could also be calculated. The crystallinities and the percentages of $\gamma$-crystalline are summarized in Table IV and Table $\mathrm{V}$ respectively.

$$
X_{c}(\%)=\frac{\sum\left(A_{\alpha \text {-form }}+A_{\gamma \text {-form }}\right)}{\sum\left(A_{\alpha \text {-form }}+A_{\gamma \text {-form }}+A_{\text {amorph }}\right)} \times 100
$$

Here $A \alpha, A \gamma$ and Aamorph are the calculated areas of the diffraction peaks for $\alpha$ crystalline, $\gamma$-crystalline and amorphous region respectively.

From the results in Table IV and Table V, it can be seen that the crystallinities of neat PA6 and PA6/m-HNTs10 gradually decrease as the cooling rate increases, which is common to the usually observed phenomenon for a crystalline polymer. In the nanocomposites of PA6/m-HNTs2 and PA6/m-HNTs5, however, an abnormal situation is observed. The crystallinities of the PA6/m-HNTs nanocomposites increase first as the 

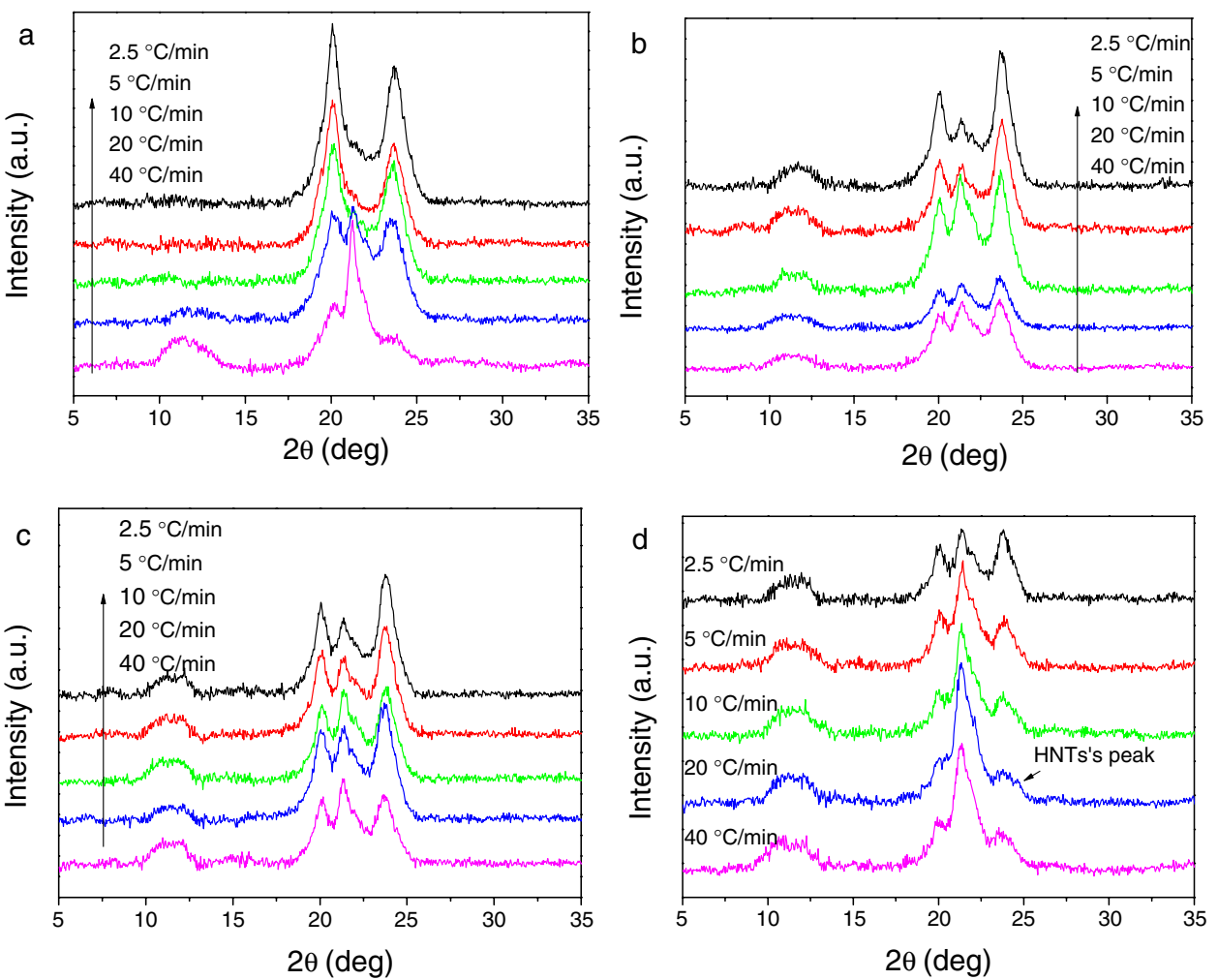

Figure 12. XRD patterns of PA6 and PA6/m-HNTs nanocomposites at various cooling rates (a) neat PA6, (b) PA6/m-HNTs2, (c) PA6/m-HNTs5, (d) $\mathrm{PA} 6 / \mathrm{m}$-HNTs10.

Table IV. Crystallinities calculated from XRD spectra of PA6 and PA6/m-HNTs nanocomposites

\begin{tabular}{cccccc}
\hline Cooling Rate & $2.5^{\circ} \mathrm{C} / \min$ & $5^{\circ} \mathrm{C} / \mathrm{min}$ & $10^{\circ} \mathrm{C} / \min$ & $20^{\circ} \mathrm{C} / \min$ & $40^{\circ} \mathrm{C} / \mathrm{min}$ \\
\cline { 1 - 4 } Sample & & & & & \\
\hline Neat PA6 & 62.85 & 60.12 & 59.46 & 55.77 & 45.46 \\
PA6/m-HNTs2 & 46.84 & 55.02 & 56.03 & 52.98 & 50.61 \\
PA6/m-HNTs5 & 50.44 & 57.15 & 52.4 & 50.56 & 47.69 \\
PA6/m-HNTs10 & 55.09 & 53.57 & 52.7 & 42.21 & 38.54 \\
\hline
\end{tabular}

cooling rate increases and then decrease as the cooling rate increases. In addition, it is noticeable that, at the same cooling rate, $\gamma$-crystal percentage increases as the m-HNTs loading increases. Although at a higher cooling rate this trend is slightly changed, we can still conclude that both high cooling rate and high $\mathrm{m}$-HNTs loading are beneficial to the formation of $\gamma$-crystal.

The dependence of crystallinity of the nanocomposites on the cooling rate could be explained below. There are two stages, i.e., nucleation and growth, during the crystallization process. On the one hand, m-HNTs in the matrix plays a role in heterogeneous nucleation, which greatly promotes the formation of the nuclei; On the other hand, because of the covalent linkages between m-HNTs and PA6 chains, the mobility of the PA6 chains around m-HNTs is severely constrained and the crystal growth is retarded. When the m-HNTs loading is relatively lower, the constraint effects of the interfacial interaction is not sufficiently high to affect the grain growth.
Table V. Values of $[\gamma /(\gamma+\alpha)] \%$ calculated from XRD spectra of PA6 and PA6/m-HNTs nanocomposites

\begin{tabular}{|c|c|c|c|c|c|}
\hline Cooling Rate & \multirow{2}{*}{$2.5^{\circ} \mathrm{C} / \mathrm{min}$} & \multirow{2}{*}{$5^{\circ} \mathrm{C} / \min$} & \multirow{2}{*}{$10^{\circ} \mathrm{C} / \mathrm{min}$} & \multirow{2}{*}{$20^{\circ} \mathrm{C} / \mathrm{min}$} & \multirow{2}{*}{$40^{\circ} \mathrm{C} / \mathrm{min}$} \\
\hline Sample & & & & & \\
\hline Neat PA6 & 7.84 & 11.01 & 12.66 & 37.05 & 55.21 \\
\hline PA6/m-HNTs2 & 15.50 & 20.99 & 35.16 & 31.84 & 36.81 \\
\hline PA6/m-HNTs5 & 19.69 & 26.25 & 32.82 & 33.23 & 42.08 \\
\hline PA6/m-HNTs10 & 31.78 & 55.53 & 61.31 & 76.55 & 73.69 \\
\hline
\end{tabular}

Consequently, as the cooling rate increases, the nucleation in the nanocomposites is accelerated and the crystallinity increases. With further high cooling rate, the time for grain growth is greatly reduced and consequently the crystallinity decreases. For PA6/m-HNTs10, although the nucleation is promoted, the grain growth is greatly restricted by the interfacial interaction and the largely decreased space due to the excellent dispersion of m-HNTs in the matrix. Consequently, the crystallization is simply controlled by crystallization time and the crystallinity of PA6/m-HNTs10 decreases monotonously as the cooling rate increases.

\section{CONCLUSIONS}

Halloysite nanotubes (HNTs) were silylated with 3-(trimethoxysilyl)propyl methacrylate to yield modified HNTs (mHNTs). The silylation process was verified to be very effective by the spectral evidences and the hydrophobicity of m-HNTs. 
The nanocomposites consisting of PA6 and m-HNTs showed significantly improved mechanical properties and heat distortion temperature. These improvements were attributed to the covalent interfacial bonding and the excellent dispersion state of m-HNTs. The nucleation effect of m-HNTs was confirmed by the decreased fold-surface free energy of the PA6/HNTs nanocomposites and evidence from the POM observation. Both high cooling rate and high m-HNTs loading favored higher $\gamma$ crystals fraction in the nanocomposites. The polymorphism of the nanocomposites was correlated to the heterogeneous nucleation effects of m-HNTs and the interfacial interactions between m-HNTs and PA6 matrix.

Acknowledgment. We are grateful for the financial support by the National Natural Science Foundation of China with grant numbers of 50603005 and 50873035 .

Received: May 3, 2009

Accepted: June 22, 2009

Published: August 5, 2009

\section{REFERENCES}

1. "Nylon Plastics Handbook," M. I. Kohan, Ed., Hanser Gardner Publications, 1995.

2. A. Usuki, Y. Kojima, M. Kawasumi, A. Okada, T. Kurauchi, and O. Kamigaito, J. Mater. Res., 8, 1179 (1993).

3. Y. Kojima, A. Usuki, Y. Kojima, M. Kawasumi, A. Okada, T. Kurauchi, and O. Kamigaito, J. Mater. Res., 8, 1185 (1993).

4. A. N. Wilkinson, Z. Man, J. L. Stanford, P. Matikainen, M. L. Clemens, G. C. Lees, and C. M. Liauw, Compos. Sci. Technol., 67, 3360 (2007).

5. A. N. Wilkinson, Z. Man, J. L. Stanford, and C. M. Liauw, Macromol. Mater. Eng., 291, 917 (2006).

6. M. Fermeglia, M. Ferrone, and S. Pricl, Fluid Phase Equilib., 212, 315 (2003)

7. T. X. Liu, I. Y. Phang, L. Shen, S. Y. Chow, and W. D. Zhang, Macromolecules, 37, 7214 (2004).

8. C. G. Zhao, G. J. Hu, R. Justice, D. W. Schaefer, S. M. Zhang, M. S. Yang, and C. C. Han, Polymer, 46, 5125 (2005).

9. W. G. Shao, Q. Wang, F. Wang, and Y. H. Chen, Carbon, 44, 2708 (2006).
10. B. L. Pan, Q. F. Yue, J. F. Ren, H. G. Wang, L. Q. Jian, J. Y. Zhang, and S. R. Yang, Polym. Test., 25, 384 (2006).

11. B. L. Pan, Q. F. Yue, J. F. Ren, H. G. Wang, L. Q. Jian, J. Y. Zhang, and S. R. Yang, J. Macromol. Sci., Part B: Phys., 45, 1025 (2006).

12. J. B. Dixon and T. R. McKee, Clays Clay Miner., 22, 127 (1974).

13. G. J. Churchman, T. J. Davy, and L. A. G. Aylmore, Clay Miner., 30, 89 (1995).

14. T. F. Bates, F. A. Hildebrand, and A. Swineford, Am. Mineral., 35, 463 (1950).

15. R. R. Price, B. P. Gaber, and Y. M. Lvov, J. Microencapsulation, 18, 713 (2001).

16. E. Joussein, S. Petit, J. Churchman, B. Theng, D. Righi, and B. Delvaux, Clay Miner., 40, 383 (2005).

17. Y. P. Ye, H. B. Chen, J. S. Wu, and L. Ye, Polymer, 48, 6426 (2007).

18. M. X. Liu, B. C. Guo, Q. L. Zou, M. L. Du, and D. M. Jia, Nanotechnology, 19, 205709 (2008).

19. M. X. Liu, B. C. Guo, M. L. Du, X. J. Cai, and D. M. Jia, Nanotechnology, 18, 455703 (2007).

20. M. L. Du, B. C. Guo, and D. M. Jia, Polym. J., 38, 1198 (2006).

21. B. C. Guo, Q. L. Zou, Y. D. Lei, M. X. Liu, and D. M. Jia, New Chem. Mater. (in Chinese) 6, 32 (2008).

22. G. Gurato, A. Fichera, F. Z. Grandi, R. Zanetti, and P. Canal, Makromol. Chem., 175, 953 (1974).

23. D. M. Lincoln, R. A. Vaia, and R. Krishnamoorti, Macromolecules, 37, 4554 (2004).

24. M. Colilla, M. Darder, P. Aranda, and E. Ruiz-Hitzky, J. Mater. Chem., 15, 3844 (2005).

25. http://riodb01.ibase.aist.go.jp/sdbs/cgi-bin/cre_index.cgi?lang=eng

26. T. X. Liu and I. Y. Phang, Macromolecules, 37, 7214 (2004).

27. Y. Kojima, A. Usuki, M. Kawasumi, A. Okada, and T. O. Kurauchi, J. Mater. Res., 8, 1185 (1993).

28. B. C. Guo, Q. L. Zou, Y. D. Lei, M. L. Du, M. X. Liu, and D. M. Jia, Thermochim. Acta, 484, 48 (2009).

29. J. D. Hoffman, G. T. Davis, and J. I. Lauritzen, in "Treatise on Solid State Chemistry,” N. B. Hannay, Ed., Plenum, New York, 1976, p 497.

30. S. Vyazovkin, J. Comput. Chem., 18, 393 (1997).

31. S. Vyazovkin, J. Comput. Chem., 22, 178 (2001).

32. D. R. Holmes, C. W. Bunn, and D. J. Smith, J. Polym. Sci., 17, 159 (1955).

33. H. Arimoto, M. Ishibashi, M. Hirai, and Y. Chatani, J. Polym. Sci., Part A: Polym. Chem., 3, 317 (1965).

34. X. H. Liu, Q. J. Wu, and L. A. Berglund, Macromol. Mater. Eng., 287, 515 (2002).

35. M. K. Akkapeddi, Presented at ANTEC 99 Conference, New York, 1999. 